Article

\title{
Fluorinated Phenylalanine Precursor Resistance in Yeast
}

\author{
Ian S. Murdoch (iD, Samantha L. Powers and Aaron Z. Welch * \\ Biomolecular Sciences Institute, Florida International University, Miami 33199, FL, USA; \\ imurd001@fiu.edu (I.S.M.); spowe009@fiu.edu (S.L.P.) \\ * Correspondence: aawelch@fiu.edu; Tel.: +1-305-919-4033
}

Received: 27 April 2018; Accepted: 4 June 2018; Published: 9 June 2018

\begin{abstract}
Development of a counter-selection method for phenylalanine auxotrophy could be a useful tool in the repertoire of yeast genetics. Fluorinated and sulfurated precursors of phenylalanine were tested for toxicity in Saccharomyces cerevisiae. One such precursor, 4-fluorophenylpyruvate (FPP), was found to be toxic to several strains from the Saccharomyces and Candida genera. Toxicity was partially dependent on $A R O 8$ and ARO9, and correlated with a strain's ability to convert FPP into 4-fluorophenylalanine (FPA). Thus, strains with deletions in ARO8 and ARO9, having a mild phenylalanine auxotrophy, could be separated from a culture of wild-type strains using FPP. Tetrad analysis suggests FPP resistance in one strain is due to two genes. Strains resistant to FPA have previously been shown to exhibit increased phenylethanol production. However, FPP resistant isolates did not follow this trend. These results suggest that FPP could effectively be used for counter-selection but not for enhanced phenylethanol production.
\end{abstract}

Keywords: fluorinated compounds; counter selection; phenylalanine; phenylethanol; yeast

\section{Introduction}

The phenylalanine biosynthetic pathway is a route to production of the high-value chemical 2-phenylethanol that is used for its rosy scent in cosmetics, foods, and cleaning supplies [1]. Phenylethanol is produced mainly by synthetic chemical processes but also naturally by yeast, as a degradation product of phenylalanine, moving through the intermediate metabolites of phenylpyruvate then phenylacetaldehyde (Figure 1). In Saccharomyces cerevisiae, one method of increasing the production of these chemicals is to select for resistance to the fluorinated phenylalanine analog 4-fluorophenylalanine (FPA) [2]. One facet of the mechanism of resistance is enhanced endogenous production of phenylalanine, thus requiring less dependence on exogenously provided phenylalanine [3]. Hence, FPA can be used to increase production of phenylethanol.

There are numerous counter-selection methods for a variety of genes. One method, the use of toxic metabolites to counter-select for auxotrophies such as uracil or tryptophan, has been a boon in yeast genetics, enabling numerous techniques such as the plasmid shuffle and Synthetic Genetic Array analysis [4-6]. These techniques rely on adding a fluorine to a precursor of the final biosynthetic pathway product, such that use of the fluorinated precursor results in a fluorinated end-product, which is toxic to the cell. There are many potential mechanisms of resistance to toxic precursors. One such mechanism is the prohibition of toxic metabolite import, as in the case of canavanine resistance via abrogation of the arginine transporter [4]. Another is to decrease endogenous production of the precursor while relying on exogenous end-product for growth, as in the case of deletion of URA3 and reliance upon external uracil for 5-fluoroorotic acid resistance. In the case of resistance to a toxic end-product, one mechanism of resistance is via increasing endogenous production of the end-product 
so as to decrease exogenous nutrient dependence, such as in the case of increased phenylalanine production in response to fluorophenylalanine [3].

We sought to identify toxic precursors to the phenylalanine biosynthetic pathway that could be used for auxotrophic counter-selection, and that may lead to increased production of phenylethanol.

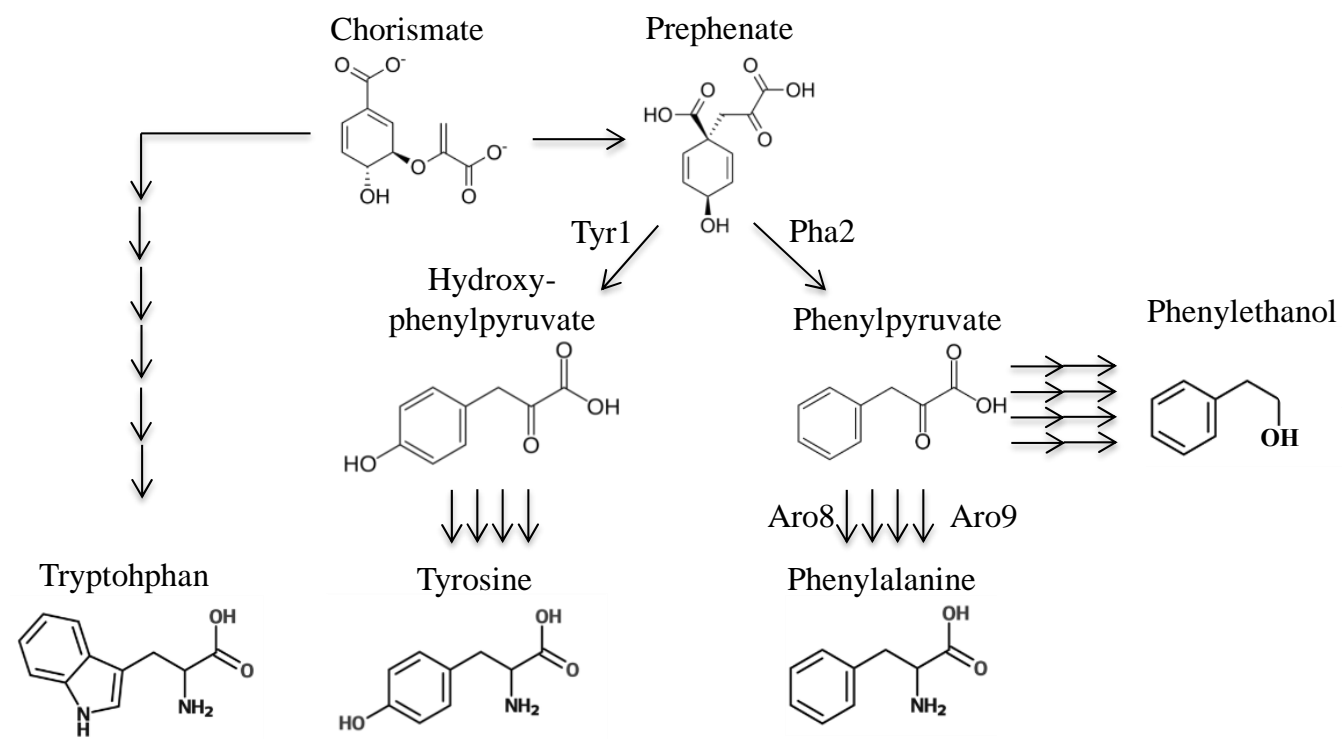

Figure 1. Diagram of the yeast pathway for biosynthesis of tryptophan, tyrosine, and phenylalanine. Number of enzymes and steps are indicated by number of arrows.

\section{Materials and Methods}

\subsection{Media and Growth Conditions}

Rich medium, or YPD, was made with BactoTM Yeast Extract at $10 \mathrm{~g} \mathrm{~L}^{-1}$ (Becton Dickinson, Franklin Lakes, NJ, USA), BactoTM Peptone at $20 \mathrm{~g} \mathrm{~L}^{-1}$ (Becton Dickinson), and dextrose at $20 \mathrm{~g} \mathrm{~L}^{-1}$ (Fisher Scientific, Waltham, MA, USA). Minimal medium contains $1.8 \mathrm{~g} \mathrm{~L}^{-1}$ yeast nitrogen base without amino acid or ammonium sulfate and $6.2 \mathrm{~g} \mathrm{~L}^{-1}$ ammonium sulphate and dextrose at $20 \mathrm{~g} \mathrm{~L}^{-1}$. Synthetic complete medium contains minimal medium with $20 \mathrm{mg} \mathrm{L}^{-1}$ of all the following compounds: uracil, lysine, tyrosine, tryptophan, phenylalanine, adenine, leucine, histidine, and methionine. For clarification, the phenylalanine-related chemicals used in this paper are described in Table 1, their molecular structures are shown in Figure 2.

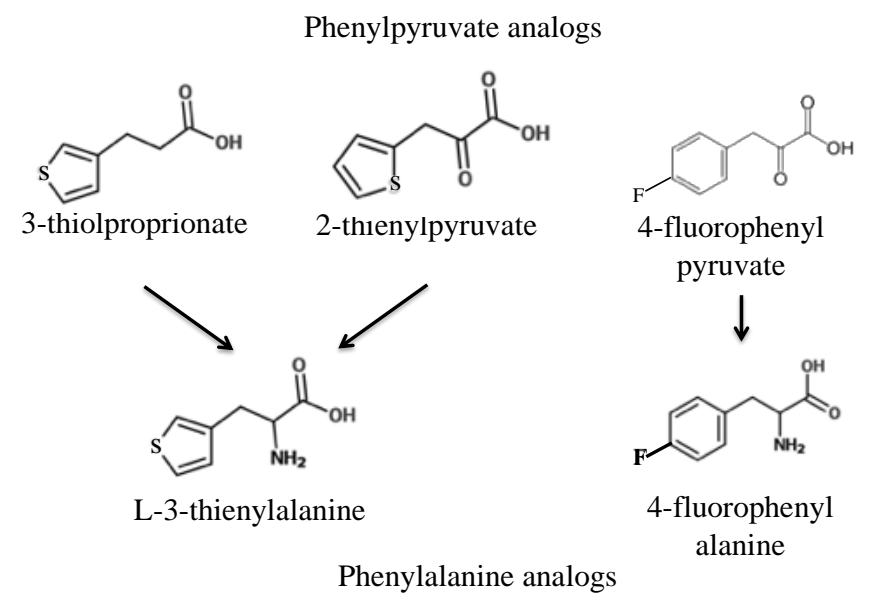

Figure 2. Analogs of phenylpyruvate and phenylalanine tested. 
Table 1. List of phenylalanine-related drugs used.

\begin{tabular}{cccc}
\hline Chemical Name & Abbreviation & CAS \# & Source \\
\hline 4-fluorophenylalanine & FPA & $1132-68-9$ & Chem-Impex \\
4-fluorophenylpyruvate & FFP & $7761-30-0$ & Enamine \\
2-thienylpyruvate & 2TP & $15504-41-3$ & Enamine \\
3-thiolproprionate & 3TP & $16378-06-6$ & Enamine \\
L-3-thienylalnine & L3T & $3685-51-6$ & Sigma \\
\hline
\end{tabular}

Chem-Impex (Wood Dale, IL, USA);Enamine (Monmouth Jct., NJ, USA); Sigma (St. Louis, MO, USA). CAS\# refers to Chemical Abstracts Service registry number for indicated compound.

Experiments using FPA, FPP, 2TP, 3TP, or L3T were performed in MM2 medium, which consists of minimal medium with $2 \mathrm{mg} \mathrm{L}^{-1}$ of all the following compounds: uracil, tyrosine, phenylalanine, leucine, histidine, and methionine. Chemicals FPA, 2TP, 3TP, or L3T were suspended in water, while FPP and FPA were suspended in dimethyl sulfoxide (DMSO).

Culture density was determined by a spectrophotometer reading of the optical density at $600 \mathrm{~nm}$, which was correlated to cell density via hemocytometer counts. Strains used are listed in Table 2. Strains mentioned with a dash (e.g., S288c-3) are derivatives of the parental strain named preceding the dash. Mutation cultures were obtained by exposure to ultraviolet light at $230 \mathrm{~nm}$ for $10 \mathrm{~s}$ that resulted in viability between $30-70 \%$ as measured by methylene blue.

Table 2. List of yeast strains used.

\begin{tabular}{|c|c|c|}
\hline Strain & Genotype & Source \\
\hline $26704 c$ & MAT $\alpha$ ura3 aro8-2 aro9-1 & [7] \\
\hline BY4700 & $M A T \alpha S 288 c$ ura3 $\Delta 0$ & ATCC \\
\hline BY4742 & 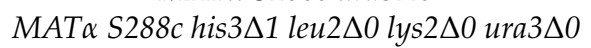 & ATCC \\
\hline A364A & MATa ade1 ade2 ura1 his7 lys2 tyr1 gal1 & ATCC \\
\hline AW051 & MATa ura3 aro8-2 aro9-1 & This study \\
\hline AW052 & MATa ura3 aro8-2 aro9-1 & This study \\
\hline AW063 & MAT $\alpha$ ade2 pha2 arg8 lys2 & This study \\
\hline AW077 & MAT $\alpha$ ade2 ura1 & This study \\
\hline AW081 & MATa ura1 his7 & This study \\
\hline AW083 & 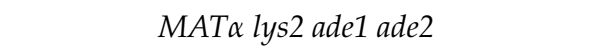 & This study \\
\hline AW102 & MATa/MAT $\alpha$ wild-type & This Study \\
\hline AW108 & MAT $\alpha$ S288c pha2::G418 & This study \\
\hline AW109 & 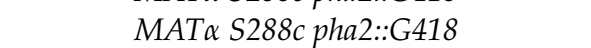 & This study \\
\hline AW258 & MAT $\alpha$ ura1 & This study \\
\hline AW259 & MAT $\alpha$ his7 & This study \\
\hline AW261 & MAT $\alpha$ met15 & This study \\
\hline IM006 & MAT $\alpha$ lys2 & This study \\
\hline Calb & Candida albicans ATCC 10231 & Gift from Darlene Miller \\
\hline Ctrop & Candida tropicalis & Gift from Darlene Miller \\
\hline S288c & 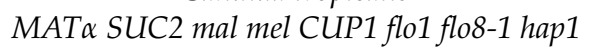 & ATCC \\
\hline SPara & lys2 Saccharomyces paradoxus & Gift from Doug Koshland \\
\hline SBaya & ura3 Saccharomyces bayanus & Gift from Doug Koshland \\
\hline
\end{tabular}

American Type Culture Collection (ATCC)

Drug resistance was measured by calculating the relative viability of cells, which is the number of viable cells resulting from a drug treated culture divided by the number of viable cells resulting from a carrier treated (water or DMSO) culture. Viability was measured as colony forming units on a petri dish by directly plating cells to a plate or by first serially diluting in water, then pinning to drug and carrier plates (frogging method) or by using the Tadpoling method, discussed below. For timecourse experiments, relative viability was calculated by using the number of viable cells at timepoint zero as the denominator in all subsequent timepoints. 
Briefly, the Tadpoling method consists of inoculating $20 \mu \mathrm{L}$ of yeast containing culture into $180 \mu \mathrm{L}$ YPD in a well of a 96-well plate and serially diluting $20 \mu \mathrm{L}$ from this well to subsequent wells [8]. This plate is incubated until individual colonies can be counted in the most dilute well, yielding a quantifiable number used to calculate cell viability in the original well by multiplying by the dilution factor.

$$
\text { hours } \times \log _{2}\left(\frac{\text { ODin }}{\text { ODfin }}\right)
$$

Growth rate was calculated using the following expression: where ODin is the initial A600, ODfin is the final A600, hours is the number of hours in between A600 readings.

\subsection{Determination of Phenylethanol and Phenylalanine}

After growth in $2 \mathrm{mg} \mathrm{L}^{-1}$ uracil, tyrosine, phenylalanine, leucine, histidine, and methionine with $1.8 \mathrm{~g} \mathrm{~L}^{-1}$ yeast nitrogen base without amino acid or ammonium sulfate with $20 \mathrm{~g} \mathrm{~L}^{-1}$ dextrose and $6 \mathrm{~g} \mathrm{~L} \mathrm{~L}^{-1}$ phenylalanine for $6-8$ days at $30^{\circ} \mathrm{C}$, culture supernatant was removed and selected ion monitoring (SIM) was performed to detect phenylethanol and phenylalanine using liquid chromatography-tandem mass spectrometry (LC-MS/MS). A gradient was run from $100 \% 50 \mathrm{mM}$ ammonium acetate to $100 \%$ methanol with $0.1 \%$ formic acid over the course of $20 \mathrm{~min}$. Phenylethanol retention time was $12.5 \mathrm{~min}$ while that of phenylalanine was 4.5 .

\subsection{Determination of Fluorophenylalanine}

Yeast samples were grown in medium containing FPP at a concentration of $200 \mathrm{mg} \mathrm{L}^{-1}$ for $16 \mathrm{~h}$ then yeast samples were resuspended in $70 \%$ ethanol, boiled for $10 \mathrm{~min}$, then the supernatant was transferred to a new tube and after evaporated in a spinning-vacuum centrifuge. Samples were fully dissolved in $1 \mathrm{~mL}$ of 3:1:1 water:acetronitrile:isopropanol, with $2 \mathrm{~min}$ of vortexing and $20 \mathrm{~min}$ sonication. The resulting solutions were $5 \times$ diluted (200/1000) in $10 \mathrm{mM}$ ammonium formate in water (mobile phase A) and was injected in the LC-MS/MS system, an AB Sciex QTRAP 5500 Triple-Quadrupole mass spectrometer, equipped with a Turbospray ESI source. A binary gradient separation program was employed using a reverse phase HPLC column (Dionex Acclaim 120 C18 Column, $250 \times 2.1 \mathrm{~mm}, 5 \mu \mathrm{m})$. Sample injection $(20 \mu \mathrm{L})$ and LC separation was performed by a Shimadzu Prominence LC-20AD Ultra-Fast Liquid Chromatograph.

\section{Results}

First, we sought to identify a toxic phenylalanine analog and work backward to design toxic phenylalanine precursors. Two promising toxic phenylalanine analogs were identified from the literature: L-3-thienylalanine (L3T), consisting of phenylalanine with the benzene ring substituted for thiophene, and fluorophenylalanine (FPA), consisting of phenylalanine with a fluorine attached to the benzene ring (Figure 2, Table 1). A $2.6 \mathrm{mg} \mathrm{L}^{-1}$ concentration of L-3-thienylalanine has been shown to inhibit growth of S. cerevisiae by 50\% [9]. First, the toxicity of L3-thienylalanine was tested by plating wild-type strains S288c and AW077 to medium with L3-thienylalanine at 0 or $300 \mathrm{mg} \mathrm{L}^{-1}$ and determining the relative viability. L3T killed all AW077 cells and nearly all of S288c cells (Figure 3a). Next the toxicity of FPA was tested by plating strains S288c and AW077 onto medium containing FPA at 0 or $300 \mathrm{mg} \mathrm{L}^{-1}$ and determining the relative viability (Figure $3 \mathrm{~b}$ ). FPA at both of these concentrations killed almost all cells of S288c and AW077. Thus, we reconfirmed that L3T and FPA are toxic to $S$. cerevisiae.

Next, we sought to design a molecule that may be enzymatically converted to a toxic phenylalanine analog. By identifying a toxic phenylalanine precursor, we could use this compound to select for strains that have mutations in the phenylalanine biosynthetic pathway. We considered several precursors of phenylalanine; chorismate (this precursor is used to synthesize not only phenylalanine, but also tryptophan, tyrosine, folate, and ubiquinone). Thus, a strain with a chorismate deletion would have numerous other auxotrophies besides phenylalanine. Next, prephenate-however, this is not 
a good candidate for this particular intervention as adding fluorine or sulfur on the cyclohexadiene ring could interfere with transformation to a benzene ring. Additionally, there seems to be at least two mechanisms to convert prephenate to phenylalanine, as we found that deletion of PHA2 does not confer complete phenylalanine requirement. To test this, strains lacking $P H A 2$ were inoculated alongside a strain with functioning PHA2 in minimal medium with or without phenylalanine added (Figure 4, Table 3). The A600 was measured over four days, and maximum growth rate (lowest doubling time) was calculated. Strains AW108 and AW109 grew much better with phenylalanine added (Table 3), showing the dependency on phenylalanine; however, they still grew over time. This is in contrast to previously described observations of strains lacking PHA2. However, it is likely that the extremely slow growth rate explains this conclusion (Figure 4) [10]. The next candidate is phenylpyruvate, which does not require modification of its benzene ring prior to becoming phenylalanine, and it is the substrate for only two essentials molecules: phenylalanine and tyrosine. Use of phenylpyruvate has one drawback: there are two primary enzymes that can complete the transamination reaction converting phenylpyruvate to phenylalanine (ARO8 and ARO9) and several others that are predicted to be capable of performing this reaction (Bat1, Bat2, His5) [11].

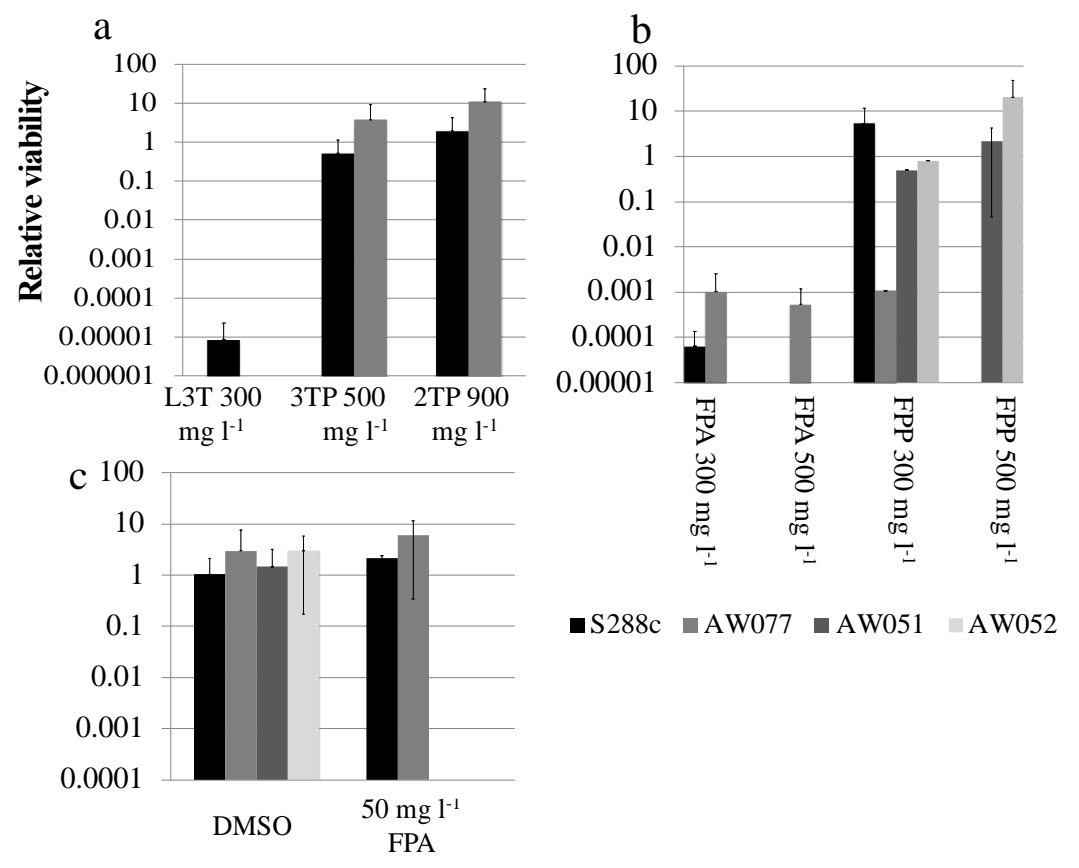

Figure 3. Viability of yeast in response to toxic phenylalanine or phenylpyruvate analogs. Cultures of yeast strains were serially diluted in water then plated onto medium containing either (a) L3T, 3TP, 2TP or (b) FPA, FPP, or (c) dimethyl sulfoxide, FPA. Relative viability was obtained by dividing the number of colony forming units (CFUs) from drug plates by CFUs from YPD plates. Plates were incubated for 2-3 days and CFUs counted. The average and standard deviation of three independent trials is shown.

Table 3. Density of two-day-old cultures.

\begin{tabular}{cccc}
\hline Strain & Genotype & $\mathbf{M M}+\mathbf{U}^{\mathbf{a}}$ & $\mathbf{M M}+\mathbf{U P}^{\mathbf{b}}$ \\
\hline AW108 & pha2 & 0.06 & 3.8 \\
AW109 & pha2 & 0.08 & 3.6 \\
AW051 & aro8 aro9 ura3 & 0.37 & 1.1 \\
AW052 & aro8 aro9 ura3 & 1.2 & 2.2 \\
BY4700 & ura3 & 3.6 & 3.7 \\
\hline
\end{tabular}

MM: Minimal Medium a Minimal medium with $20 \mathrm{mg} \mathrm{L}^{-1}$ uracil, $^{\mathrm{b}}$ Minimal medium with $20 \mathrm{mg} \mathrm{L}{ }^{-1}$ of uracil and phenylalanine. 


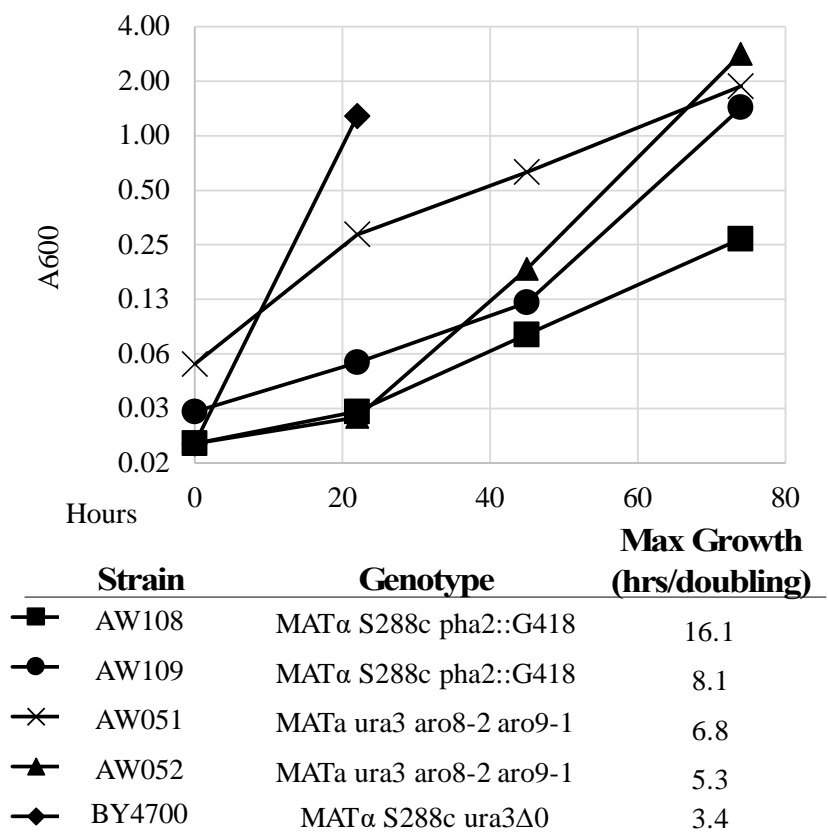

Figure 4. Growth rate of strains in medium lacking phenylalanine. The indicated strains were grown overnight in synthetic complete medium then washed and inoculated to minimal medium with uracil and rotated at $23{ }^{\circ} \mathrm{C}$. The A600 was measured over subsequent hours. Max growth was the lowest growth rate calculated for all points tested.

This presents a problem for selection, as strains would need to have deletions in two genes, rather than just one, in the case of uracil (URA3), lysine (LYS2), or arginine (CAN1) [6,12,13]. To test if ARO8 and ARO9 were required for phenylalanine production, strains lacking ARO8 and ARO9 (AW051, AW052) were constructed and inoculated alongside a wild-type strain in minimal medium with or without phenylalanine added (Figure 4, Table 3).

The A600 was measured over four days, and maximum growth rate (lowest doubling time) was calculated. Strains AW051 and AW052 grew much better with phenylalanine added (Table 3), showing their dependency on phenylalanine. However, they still grew over time. These strains do grow faster than the PHA2 mutants but are not completely rescued solely by phenylalanine, as they also require tyrosine, hence the lower A600 on the second day of growth with phenylalanine added.

Furthermore, to verify there were no residual nutrients in the minimal medium, strains with various individual auxotrophies were inoculated to minimal medium and were found to not grow (Table 4).

Table 4. Density of three-day-old cultures.

\begin{tabular}{cccc}
\hline Strain & Genotype & MM & $\begin{array}{c}\text { MM + Aux } \\
\text { a }\end{array}$ \\
\hline AW108 & pha2 & 3.0 & 5.8 \\
AW109 & pha2 & 2.8 & 5.6 \\
AW258 & ura1 & 0.1 & 3.6 \\
AW259 & his7 & 0.04 & 0.3 \\
AW261 & met15 & 0.08 & 5.2 \\
IM006 & lys2 & 0.07 & 1.4 \\
\hline
\end{tabular}

a Minimal medium with $20 \mathrm{mg} \mathrm{L}^{-1}$ of auxotrophy required for strain.

Nonetheless, multiple enzymes producing phenylalanine could be viewed as a boon, as cells are less likely to develop spontaneous resistance to a toxic phenylpyruvate analog, as they would have to delete multiple processing enzymes. Thus we set out to design phenylpyruvate-like derivatives 
that may be catalytically converted into toxic phenylalanine analogs so that these can be used for counter-selection of phenylalanine auxotrophies.

The first two phenylpyruvate-like derivatives of L-3-thienylalanine tested were 3-thiolproprionate (3TP) and 2-thienylpyruvate (2TP). Wild-type yeast strains S288c and AW077 were pinned to plates containing these analogs at $500 \mathrm{mg} \mathrm{L}^{-1}$ and $900 \mathrm{mg} \mathrm{L}^{-1}$, respectively, and relative viability was calculated (Figure 3a). Despite higher concentrations of the phenylpyruvate derivatives compared to L3T, S288c and AW077 strains exhibited no decrease in viability on 3TP and 2TP containing medium. Thus, while the phenylalanine analog was toxic to these strains, the phenylpyruvate analogs were not. Differences in toxicity could be due to adsorption to the cell wall, decreased uptake, or incompatibility with enzymes.

The toxicity of the FPA derivative fluorophenylpyruvate (FPP) was next examined. Strains AW077 and S288c were serially diluted in water, then pinned to plates containing $0,300 \mathrm{mg} \mathrm{L}^{-1}$, or $500 \mathrm{mg} \mathrm{L}^{-1}$ FPP and relative viability was calculated (Figure 3b). At $300 \mathrm{mg} \mathrm{L}^{-1}$ FPP strain S288c showed mild growth inhibition but no lethality, while strain AW077 was more growth-inhibited. At $500 \mathrm{mg} \mathrm{L}^{-1}$, no growth was detected from S288c and AW077 strains (Figure 3b). These results indicate that the phenylpyruvate analog FPP is toxic to S288c and AW077 strains.

Phenylpyruvate is converted to phenylalanine primarily by the proteins Aro8p and Aro9p. However, there are other proteins that can catalyze this reaction. To test if the mechanism of FPP toxicity was due to its conversion to FPA, the toxicity of FPP was tested on strains lacking genes ARO8 and ARO9 (Figure 3b). Strains lacking ARO8 and ARO9 (AW051, AW052) are able to grow on this medium, showing approximately equal growth on YPD. However, these strains are hypersensitive to FPA, which may reflect the cells increased import of phenylalanine, due to a phenylalanine auxotrophy (Figure 3c). This result suggests that $A R O 8$ and $A R O 9$ are primarily responsible for affecting the toxicity of FPP.

For FPP to be of use as an auxotrophic marker, the natural resistance for it should be low. For example, the frequency of spontaneous resistance to 5-fluoroanthranilic acid or 5-fluoroorotic acid, for selection of tryptophan or uracil auxotrophs, respectively, was $6 \times 10^{-7}$ [5]. To test the natural resistance frequency of S288c and AW077 strains to FPP, we plated $5 \times 10^{6}$ cells on plates containing $500 \mathrm{mg} \mathrm{L}^{-1} \mathrm{FPP}$. We found no resistant cells grew on this medium, indicating natural resistance of wild-type strains to FPP is below a frequency of $2 \times 10^{-7}$. As a comparison to this, we also plated these strains to medium containing $500 \mathrm{mg} \mathrm{L}^{-1}$ FPA. Strains S288c and AW077 formed 240 and 86 colonies giving a frequency of resistance of $3 \times 10^{-5}$ and $5 \times 10^{-5}$, respectively. This indicates that the natural frequency of resistance to FPP is quite low, while that of FPA is higher. This indicates that resistance to FPP toxicity requires either more mutations or more specific mutations, as compared to FPA, and that perhaps these molecules do not induce toxicity via the same mechanism.

To further investigate the difference between FPP and FPA resistance, strains that were FPA resistant were tested for FPP resistance. Isolates that were resistant to FPA were plated onto medium with or without FPP and relative viability was calculated (Figure 5). Three isolates derived from strain AW083 and one from AW077 showed resistance to FPA, but no resistance to FPP. This indicates that FPA resistance is not sufficient for FPP resistance. However, an isolate from a wild-type yeast wine strain (AW102) and an S288c mutant isolate (S288c-3) were recovered that showed resistance to FPP and FPA. When the strain S288c-3 was mated to a non-resistant strain (AW081) and sporulated, the resulting haploid segregants generally showed a pattern of 1:3 resistance to FPP, while resistance to FPA showed a pattern of 2:2 (Table 5).

Table 5. Tetrad analysis of FPP and FPA resistance.

\begin{tabular}{ccc}
\hline Segregation & FPA Resistance & FPP Resistance \\
\hline $2: 2$ & 20 & 5 \\
$1: 3$ & 6 & 21 \\
\hline
\end{tabular}




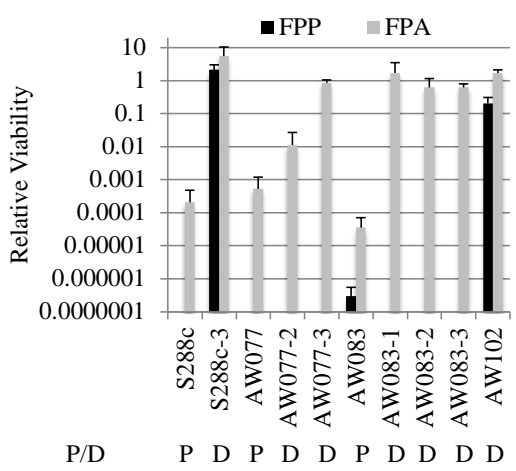

Figure 5. Viability of selected strains in response to FPP or FPA. Cultures of parental and derivative yeast strains were serially diluted in water then plated onto medium containing either $500 \mathrm{mg} \mathrm{L}^{-1}$ of FPA, FPP. Plates were incubated for 2-3 days and colony forming units (CFUs) counted. Relative viability was obtained by dividing the number of CFUs from drug plates by CFUs from YPD plates. $\mathrm{P}$ indicates the parental strain while $\mathrm{D}$ indicates a derivative of that strain. The average and standard deviation of three independent trials is shown.

A predominance of 1:3 segregation (tetratype) indicates that two genes are segregating independently, following the pattern of 1:1:4 PD:NPD:TT, while a 2:2 pattern indicates a single gene. All haploid segregants that were resistant to FPP were also resistant to FPA. The haploid segregants that were only resistant to FPA generally showed slow growth on FPA. One interpretation of these results is that detoxification of FPP requires the activity of two genes, either of which will suffice to detoxify FPA.

To determine if cells converted FPP into FPA or some other compound, we evaluated the presence of FPA in cells treated with FPP. Strains were inoculated to medium containing FPP and allowed to incubate for $>12 \mathrm{~h}$, enzymatically digested to recover individual amino acids, and then tested for the presence of FPA using LC-MS/MS (Figure 6). Strains sensitive to FPP, AW077, and S288c were observed to accumulate the highest amounts of FPA, while the less sensitive strain AW051 accumulated less. The modest decrease in FPP conversion of AW051 is not too surprising, as there are other enzymes capable of converting phenylpyruvate to phenylalanine (Bat1, Bat2, His5), and this strain is capable of growth without phenylalanine supplementation. Strains S288c-3 and AW102, both of which are resistant to FPP, had the lowest levels of FPA. This suggests that their mechanism of resistance involves decreased conversion of FPP to FPA. Thus cells can convert FPP into FPA, and this may be the cause of some of the toxicity of FPP.

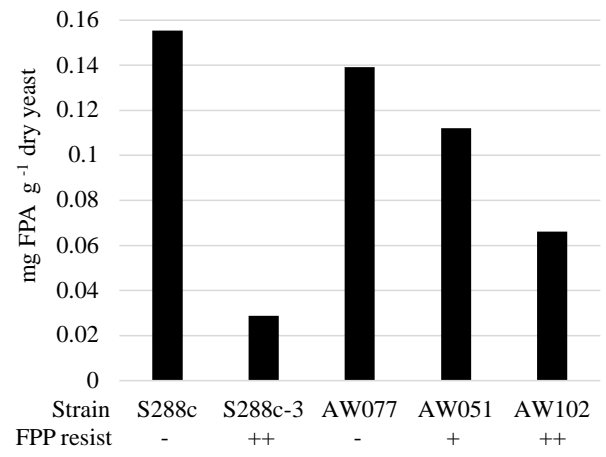

Figure 6. FPP is converted by cells to FPA. Yeast cells were inoculated to Synthetic Complete medium overnight at $30^{\circ} \mathrm{C}$, then inoculated to MM2 medium with $200 \mathrm{mg} \mathrm{L}^{-1} \mathrm{FPP}$ and incubated at $30{ }^{\circ} \mathrm{C}$ for $16 \mathrm{~h}$. Cultures were isolated, dried, weighed, and analyzed for the quantity of FPA. FPP resistance is indicated below each strain corresponding to a qualitative measure of their resistance to FPP with signifying little or no resistance, + indicating moderate resistance, and ++ indicating strong resistance. 
For FPP to be an effective selective agent, it should exert its toxic effect amongst diverse genre of yeast. To test the evolutionary conservation of FPP toxicity, strains from diverse species such as Candida tropicalis, Candida albicans, Saccharomyces bayanus, and Saccharomyces paradoxus were exposed to 0 or $500 \mathrm{mg} \mathrm{L}^{-1} \mathrm{FPP}$ or FPA and their viability was measured over several days, and relative viabilities were calculated (Figure 7). To test these strains, the FPP and FPA sensitivity test was performed by inoculating cells into media with said compounds and evaluating culture viability using the Tadpoling method [8]. All the strains tested retained viability or slightly increased in cell number when inoculated into media without FPP or FPA, except for AW083. When inoculated to media containing FPA, C. tropicalis exhibited high resistance while most of the other strains lost viability over the course of four days. Both C. albicans and S. bayanus exhibited enhanced sensitivity to FPA, as these lost almost all culture viability within one day. Surprisingly, in media containing FPP, all strains showed greater sensitivity than in FPA and lost at least four orders of magnitude culture viability within two days. This result would suggest that FPP may be transported more effectively than FPA, or that FPP can exert its toxicity through a mechanism distinct from that of FPA. S.paradoxus and AW051 (aro8-2 aro9-1) showed the least sensitivity to FPP of all the strains tested, but even these cultures succumbed after four days. This data shows that FPP can act through diverse yeast genre to exert its toxic effect and may be a more effective fungicide than FPA.
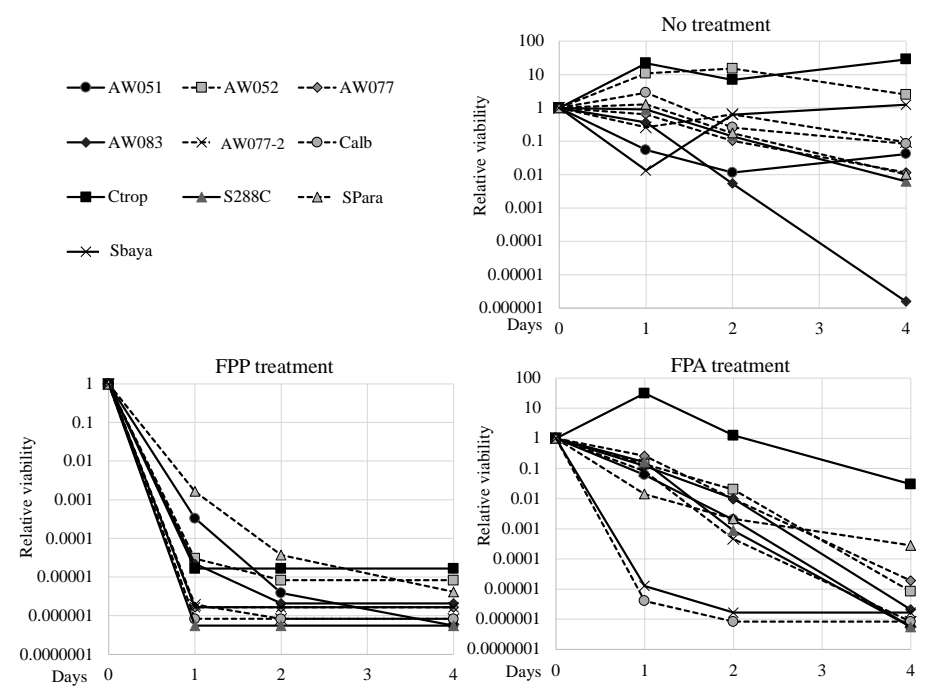

Figure 7. Toxicity of FPP and FPA are well conserved. Yeast cells were inoculated to Synthetic Complete medium overnight, then inoculated to MM2 medium with $500 \mathrm{mg} \mathrm{L}^{-1} \mathrm{FPP}$, FPA, or no drug, and incubated at $37^{\circ} \mathrm{C}$ in an immobile 96-well plate. Culture viability was assessed over time using the Tadpoling method. Relative viability was assessed by dividing the number of CFUs on day $0,1,2$, or 5 by the number of CFUs on day 0 . The average of three independent trials is shown.

One potential mechanism of FPP toxicity is to affect the mitochondria. Phenylpyruvate acts as an inhibitor of pyruvate transport into the mitochondria where pyruvate would normally be decarboxylated to acetate [14]. Indeed, accumulation of phenylpyruvate in phenylketonuria patients becomes toxic as it may disrupt oxidative metabolism by preventing normal pyruvate movement into the mitochondria. To test if phenylpyruvate alone may exert a toxic effect on yeast, exponential phase cells were inoculated to $500 \mathrm{mg} \mathrm{L}^{-1}$ phenylpyruvate and culture viability was measured over several days using the Tadpoling method. The ratio of CFUs of cells exposed to phenylpyruvate with control cells was calculated over the course of several days (Figure 8). There were no significant $(p<0.05)$ deviations from the ideal 1 ratio for all strains tested, indicating that phenylpyruvate does not significantly affect culture viability. Nonetheless, there were notable deviations in decreased 
viability of S288c cells exposed to phenylpyruvate and an increase in viability of AW052 cells exposed to phenylpyruvate. Thus, it is likely that fluorine is required for fluorophenylalanine toxicity.

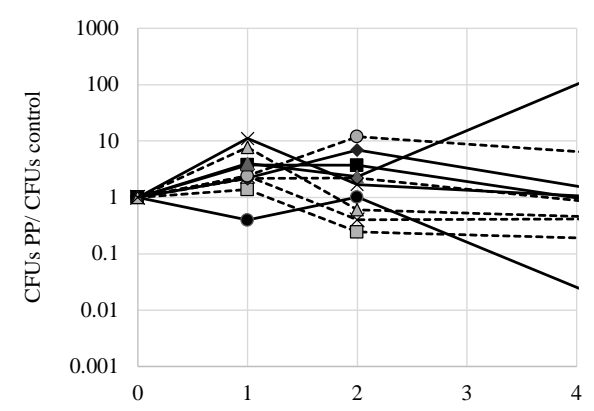

Figure 8. Phenylpyruvate does not affect culture viability. Yeast cells were inoculated to Synthetic Complete medium overnight, then inoculated to MM2 medium with $500 \mathrm{mg} \mathrm{L}^{-1}$ phenylpyruvate, or no drug, and incubated at $37^{\circ} \mathrm{C}$ in an immobile 96-well plate. Culture viability was assessed over time using the Tadpoling method. To calculate the CFUs PP/CFUs control, the number of CFUs in phenylpyruvate treated cultures was divided by the number of CFUs in the untreated culture for each day. The average of three independent trials is shown. Markers are the same as used in Figure 7.

In S. cerevisiae, one method of increasing the production of the valuable chemical phenylethanol is to select for resistance to FPA [2]. We wanted to test whether FPA resistant strains obtained herein would produce high amounts of phenylethanol. To do this, we inoculated strains into medium containing phenylalanine as the source of nitrogen and measured the phenylethanol and phenylalanine concentration in the medium after three days of growth using GC-MS (Figure 9A).
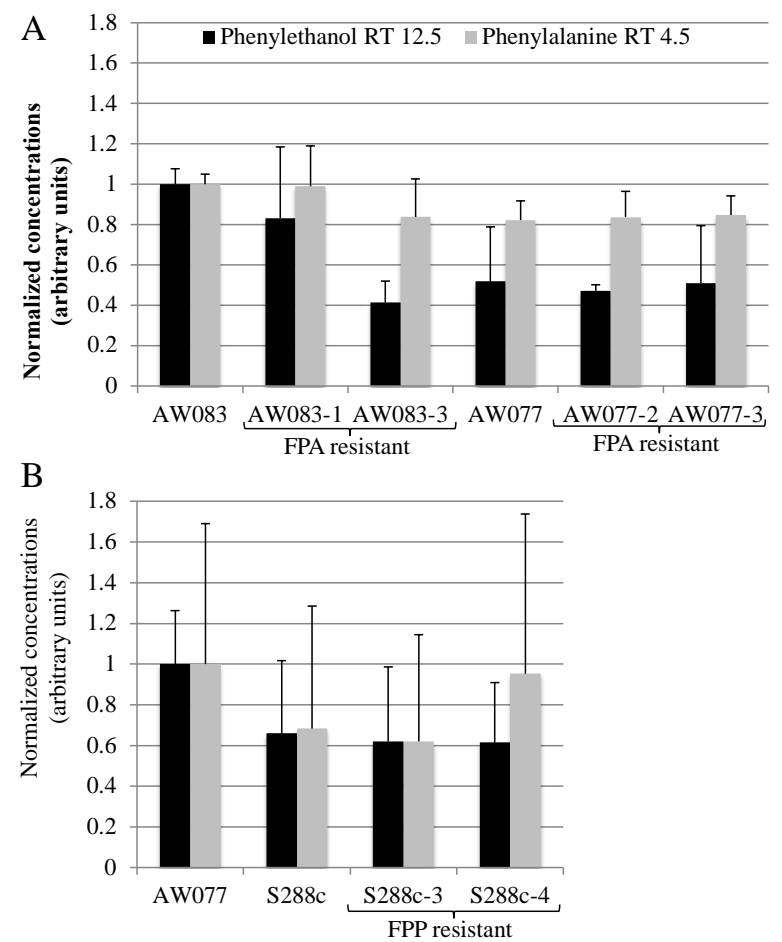

Figure 9. FPP or FPA resistance is not sufficient for increased phenylethanol production. Strains were incubated in medium containing phenylalanine as the source of nitrogen for three days, and then phenylethanol and phenylalanine concentration in the medium were measured using GC-MS. Each strain's production of phenylethanol or phenylalanine was normalized to 1 using (A) AW083 strain or (B) AW077 strain. The average and standard deviation of three independent trials is shown. 
Resistance to FPA did not induce higher phenylethanol production in the four strains that were tested. This is not surprising, as previously, the frequency of FPA-resistant mutants with high levels of phenylethanol production was approximately 20\% [15]. Thus, if we had tested five or more isolates we may have expected to obtain at least one. However, because of the low frequency of obtaining FPA-resistant strains we did not pursue this further. Because we had seen that FPP resistance was more difficult to attain than FPA, we predicted that these mutants may produce phenylalanine at a greater rate than FPA-resistant mutants and thus may have higher phenylethanol production. However, upon testing FPP-resistant strains, no increase in phenylethanol production was noted (Figure 9B). Thus, FPA and FPP resistance is not sufficient to induce high levels of phenylethanol production.

\section{Discussion}

Here, we report that FPP can act as a toxic agent in diverse strains of yeast, and this effect is at least partially mediated by ARO8 and ARO9 conversion to FPA. Whether FPP exerts its toxic effect solely by conversion to FPA or via another route rquires further investigation. FPP exhibited higher toxicity than FPA, suggesting a separate mechanism of toxicity, however this could be due to enhanced adsorption to cell wall, decreased cell uptake, or other enzymatic activities that decrease the effective concentration of FPA.

The mechanism of FPP toxicity still remains somewhat of a mystery. It is possible that there is preferred transport of phenylpyruvate over phenylalanine although evidence for this is absent. Another hypothesis is that FPP is converted into some other compound that FPA is not, such as fluoro-phenylethanol. Phenylalanine degradation follows this pathway: phenylalanine $>$ phenylpyruvate $>$ phenylacetaldehyde $>$ phenylethanol. Phenylpyruvate is one enzymatic step closer to phenylethanol, and it is possible the fluorine moiety decreases its ability to convert to fluoro-phenylalanine, but instead allows it to become fluoro-phenylethanol. Phenylethanol has been shown to be toxic to C. albicans at $20 \mathrm{mg} \mathrm{L}^{-1}$ [16].

We observed differences in the sensitivity of strains to FPP and FPA depending on the method of delivery-solid or liquid medium. Phenylpyruvate shows increased solubility relative to phenylalanine (112 $\mathrm{g} \mathrm{L}^{-1}$ versus $27 \mathrm{~g} \mathrm{~L}^{-1}$ ) that may explain its increased toxicity in liquid medium [17]. This observation may be explained by the increased exposure of cells to all toxic molecules in a liquid, as opposed to a solid medium in which cells are only exposed to toxins within close physical proximity. Previous groups have reported similar enhanced drug potency in liquid media [18].

The effect of fluorinated phenylalanine metabolites has been observed previously in other fungal species. Fluorophenylalanine at concentrations as low as $2.5 \mathrm{mM}$ can inhibit growth of fungal species Cladosporium cucumerinum and Colletotrichum lagenarium [19]. We found that FPP was toxic to a variety of yeast species at $2.74 \mathrm{mM}$ indicating that it is a more potent fungal inhibitor than FPA.

Interestingly, we also found that $P H A 2$ was not required for slow growth. This is in contrast to several other works that indicate that $P H A 2$ causes an absolute requirement for phenylalanine in the medium $[10,20]$. However, strain growth is typically assayed over a period of up to three days on solid medium, while in our studies, liquid medium was used which allows for faster growth. PHA2 converts prephenate to phenylpyruvate, but an alternative method to converting prephenate to phenylalanine is via the arogenate dehydratase enzyme. This enzyme has not been reported in S. cerevisiae. The closest species having a putative arogenate dehydratase is Neurospora crassa, which shares the same phylum with $S$. cerevisiae. Further investigation is needed to determine the mechanism of slow phenylalanine generation in strains lacking PHA2.

These observations should be helpful in better understanding phenylalanine metabolism and may be of interest in identifying potential secondary targets of FPP.

Author Contributions: Conceptualization, A.Z.W.; Methodology, A.Z.W.; Validation, A.Z.W. S.P., and I.M.; Formal Analysis, A.Z.W.; Investigation, A.W. S.P., and I.M.; Writing-Original Draft Preparation, A.Z.W.; Writing-Review \& Editing, A.Z.W. S.P., and I.M.; Visualization, A.Z.W.; Supervision, A.Z.W.; Project Administration, A.Z.W.; Funding Acquisition, A.Z.W. 
Funding: This research and APC were funded by U.S. Department of Agriculture grant NIFA-AFRI 2014-67004-21777.

Acknowledgments: The authors would like to thank Antonio Urrestarazu for the kind gift of strain 26704c. The authors would like to thank Darlene Miller for Candida albicans and Candida tropicalis strains. The authors would like to thank Doug Koshland for the kind gifts of Saccharomyces bayanus and Saccharomyces paradoxus strains. The authors would like to thank Stephanie How for her research contributions.

Conflicts of Interest: The authors declare no conflict of interest. The founding sponsors had no role in the design of the study; in the collection, analyses, or interpretation of data; in the writing of the manuscript, and in the decision to publish the results.

\section{References}

1. Etschmann, M.; Bluemke, W.; Sell, D.; Schrader, J. Biotechnological production of 2-phenylethanol. Appl. Microbiol. Biotechnol. 2002, 59, 1-8. [PubMed]

2. Akita, O.; Ida, T.; Obata, T.; Hara, S. Mutants of Saccharomyces cerevisiae producing a large quantity of $\beta$-phenethyl alcohol and $\beta$-phenethyl acetate. J. Ferment. Bioeng. 1990, 69, 125-128. [CrossRef]

3. Fukuda, K.; Watanabe, M.; Asano, K. Altered regulation of aromatic amino acid biosynthesis in $\beta$-phenylethyl-alcohol-overproducing mutants of sake yeast Saccharomyces cerevisiae. Agric. Biol. Chem. 1990, 54, 3151-3156. [CrossRef]

4. Forsburg, S.L. The art and design of genetic screens: Yeast. Nat. Rev. Genet. 2001, 2, 659-668. [CrossRef] [PubMed]

5. Toyn, J.H.; Gunyuzlu, P.L.; Hunter White, W.; Thompson, L.A.; Hollis, G.F. A counterselection for the tryptophan pathway in yeast: 5-fluoroanthranilic acid resistance. Yeast 2000, 16, 553-560. [CrossRef]

6. Boeke, J.D.; La Croute, F.; Fink, G.R. A positive selection for mutants lacking orotidine-5'-phosphate decarboxylase activity in yeast: 5-fluoro-orotic acid resistance. Mol. Gen. Genet. MGG 1984, 197, 345-346. [CrossRef] [PubMed]

7. Iraqui, I.; Vissers, S.; Cartiaux, M.; Urrestarazu, A. Characterisation of Saccharomyces cerevisiae ARO8 and ARO9 genes encoding aromatic aminotransferases I and II reveals a new aminotransferase subfamily. Mol. Gen. Genet. MGG 1998, 257, 238-248. [CrossRef] [PubMed]

8. Welch, A.Z.; Koshland, D.E. A simple colony-formation assay in liquid medium, termed tadpoling, provides a sensitive measure of Saccharomyces cerevisiae culture viability. Yeast 2013, 30, 501-509. [CrossRef] [PubMed]

9. Dittmer, K. The Synthesis and Microbiological Properties of $\beta$-3-Thienylalanine, a New anti-Phenylalanine. J. Am. Chem. Soc. 1949, 71, 1205-1207. [CrossRef] [PubMed]

10. Bross, C.D.; Corea, O.R.A.; Kaldis, A.; Menassa, R.; Bernards, M.A.; Kohalmi, S.E. Complementation of the pha2 yeast mutant suggests functional differences for arogenate dehydratases from Arabidopsis thaliana. Plant Physiol. Biochem. 2011, 49, 882-890. [CrossRef] [PubMed]

11. SRI International Yeast Pathways Database Website Home. Available online: https:/ / pathway.yeastgenome. org / (accessed on 1 May 2018).

12. Chattoo, B.B.; Sherman, F.; Azubalis, D.A.; Fjellstedt, T.A.; Mehnert, D.; Ogur, M. Selection of lys2 mutants of the yeast Saccharomyces cerevisiae by the utilization of $\alpha$-aminoadipate. Genetics 1979, 93, 51-65. [PubMed]

13. Whelan, W.L.; Gocke, E.; Manney, T.R. The CAN1 locus of Saccharomyces cerevisiae: Fine-structure analysis and forward mutation rates. Genetics 1979, 91, 35-51. [PubMed]

14. Halestrap, A.P.; Brand, M.D.; Denton, R.M. Inhibition of mitochondrial pyruvate transport by phenylpyruvate and $\alpha$-ketoisocaproate. Biochim. Biophys. Acta (BBA) Biomembr. 1974, 367, 102-108. [CrossRef]

15. Fukuda, K.; Watanabe, M.; Asano, K.; Ouchi, K.; Takasawa, S. Isolation and genetic study of p-fluoro-dl-phenylalanine-resistant mutants overproducing $\beta$-phenethyl-alcohol in Saccharomyces cerevisiae. Curr. Genet. 1991, 20, 449-452. [CrossRef] [PubMed]

16. Lingappa, B.T.; Prasad, M.; Lingappa, Y.; Hunt, D.F.; Biemann, K. Phenethyl alcohol and tryptophol: Autoantibiotics produced by the fungus Candida albicans. Science 1969, 163, 192-194. [CrossRef] [PubMed]

17. Wishart, D.S.; Feunang, Y.D.; Marcu, A.; Guo, A.C.; Liang, K.; Vázquez-Fresno, R.; Sajed, T.; Johnson, D.; Li, C.; et al. HMDB 4.0—The Human Metabolome Database for 2018. Nucleic Acids Res. 2018, 608-617. [CrossRef] [PubMed] 
18. Smith, A.M.; Ammar, R.; Nislow, C.; Giaever, G. A survey of yeast genomic assays for drug and target discovery. Pharmacol. Ther. 2010, 127, 156-164. [CrossRef] [PubMed]

19. Van Andel, O.M. Fluorophenylalanine as a systemic fungicide. Nature 1962, 194. [CrossRef]

20. Ma, Y.; Sugiura, R.; Saito, M.; Koike, A.; Sio, S.O.; Fujita, Y.; Takegawa, K.; Kuno, T. Six new amino acid-auxotrophic markers for targeted gene integration and disruption in fission yeast. Curr. Genet. 2007, 52, 97-105. [CrossRef] [PubMed]

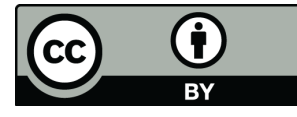

(C) 2018 by the authors. Licensee MDPI, Basel, Switzerland. This article is an open access article distributed under the terms and conditions of the Creative Commons Attribution (CC BY) license (http://creativecommons.org/licenses/by/4.0/). 